\title{
Effectiveness of adaptive silverware on range of motion of the hand
}

Susan S. McDonald, David Levine, Jim Richards, Lauren Aguilar

Background: Hand function is essential to a person's self-efficacy and greatly affects quality of life. Adapted utensils with handles of increased diameters have historically been used to assist individuals with arthritis or other hand disabilities for feeding, and other related activities of daily living. To date, minimal research has examined the biomechanical effects of modified handles, or quantified the differences in ranges of motion (ROM) when using a standard versus a modified handle. The aim of this study was to quantify the ranges of motion (ROM) required for a healthy hand to use different adaptive spoons with electrogoniometry for the purpose of understanding the physiologic advantages that adapted spoons may provide patients with limited ROM. Methods: Hand measurements included the distal interphalangeal joint (DIP), proximal interphalangeal joint (PIP), and metacarpophalangeal joint (MCP) for each finger and the interphalangeal (IP) and MCP joint for the thumb. Participants were 34 females age 18-30 (mean age 20.38 \pm 1.67 ) with no previous hand injuries or abnormalities. Participants grasped spoons with standard handles, and spoons with handle diameters of $3.18 \mathrm{~cm}$ (1.25 inch), and $4.45 \mathrm{~cm}$ (1.75 inch). ROM measurements were obtained with an electrogoniometer to record the angle at each joint for each of the spoon handle sizes. Results: A $3 \times 3 \times 4$ repeated measures ANOVA (Spoon handle size by Joint by Finger) found main effects on ROM of Joint $\left(F(2,33)=318.68\right.$, Partial $\left.\eta^{2}=.95, p<.001\right)$, Spoon handle size $(F(2,33)=598.73$, Partial $\left.\eta^{2}=.97, p<.001\right)$, and Finger $\left(F(3,32)=163.83\right.$, Partial $\left.\eta^{2}=.94, p<.001\right)$. As the spoon handle diameter size increased, the range of motion utilized to grasp the spoon handle decreased in all joints and all fingers $(p<0.01$ ). Discussion: This study confirms the hypothesis that less range of motion is required to grip utensils with larger diameter handles, which in turn may reduce challenges for patients with limited ROM of the hand. 
4 Susan McDonald Ed.D, OTR/L; David Levine PT, PhD, DPT, OCS; Jim Richards BEng, MSc, $5 \quad \mathrm{PhD}$, Lauren Aguilar, BS

6

7 From the Department of Occupational Therapy (McDonald), The University of Tennessee at

8 Chattanooga, 615 McCallie Avenue, Chattanooga, TN 37403, USA; the Department of Physical

9 Therapy (Levine), The University of Tennessee at Chattanooga, 615 McCallie Avenue,

10 Chattanooga, TN 37403, USA; the Allied Health Research unit, University of Central

11 Lancashire, UK (Richards); and the Honors College, The University of Tennessee at

12 Chattanooga, 615 McCallie Avenue, Chattanooga, TN 37403, USA

13

14 Supported by a Provost student research award from The University of Tennessee at

15 Chattanooga.

16

17 Address Correspondence to Dr. David Levine, email: David-Levine@utc.edu 18

\section{Effectiveness of Adaptive Silverware on Range of Motion of the Hand}




\section{Abstract}

20 Background: Hand function is essential to a person's self-efficacy and greatly affects quality of

21 life. Adapted utensils with handles of increased diameters have historically been used to assist

22 individuals with arthritis or other hand disabilities for feeding, and other related activities of

23 daily living. To date, minimal research has examined the biomechanical effects of modified

24 handles, or quantified the differences in ranges of motion (ROM) when using a standard versus a 25 modified handle. The aim of this study was to quantify the ranges of motion (ROM) required for 26 a healthy hand to use different adaptive spoons with electrogoniometry for the purpose of 27 understanding the physiologic advantages that adapted spoons may provide patients with limited 28 ROM.

29 Methods: Hand measurements included the distal interphalangeal joint (DIP), proximal 30 interphalangeal joint (PIP), and metacarpophalangeal joint (MCP) for each finger and the 31 interphalangeal (IP) and MCP joint for the thumb. Participants were 34 females age 18-30 (mean 32 age $20.38 \pm 1.67$ ) with no previous hand injuries or abnormalities. Participants grasped spoons 33 with standard handles, and spoons with handle diameters of $3.18 \mathrm{~cm}$ (1.25 inch), and $4.45 \mathrm{~cm}$

34 (1.75 inch). ROM measurements were obtained with an electrogoniometer to record the angle at 35 each joint for each of the spoon handle sizes.

36 Results: A 3 × 3 × 4 repeated measures ANOVA (Spoon handle size by Joint by Finger) found 37 main effects on ROM of Joint $\left(F(2,33)=318.68\right.$, Partial $\left.\eta^{2}=.95, p<.001\right)$, Spoon handle size $(F$ $(2,33)=598.73$, Partial $\left.\eta^{2}=.97, p<.001\right)$, and Finger $\left(F(3,32)=163.83\right.$, Partial $\eta^{2}=.94, p<$

$39.001)$. As the spoon handle diameter size increased, the range of motion utilized to grasp the 40 spoon handle decreased in all joints and all fingers $(\mathrm{P}<0.01)$.

41 Discussion: This study confirms the hypothesis that less range of motion is required to grip 
42 utensils with larger diameter handles, which in turn may reduce challenges for patients with

43 limited ROM of the hand.

Introduction

46 Adaptive equipment is used by approximately 23 percent of older adults in the United States,

47 indicating the importance of validating the efficacy and effectiveness of these assistive devices

48 for optimal and appropriate evidence-based prescription (Kraskowsky \& Finlayson 2001). Hand

49 impairment can inhibit or reduce functional ability to perform many activities of daily living

50 such as dressing, bathing, eating, and other self-care. It has been previously reported that the use

51 of traditional utensils to feed oneself can be difficult and/or painful with impaired hand function

52 (Brach et al. 2002). Objective assessment of hand joint range of motion (ROM) required for

53 functional activities can be valuable in prescribing adaptive equipment for individuals with

54 impairments. A person with normal hand ROM should not feel discomfort in performing tasks

55 such as gripping a standard sized eating utensil; the same task, however, can be difficult if hand

56 range of motion is limited due to either injury or disability. Examples of conditions that

57 commonly affect hand ROM include stroke, osteoarthritis, rheumatoid arthritis, and cerebral

58 palsy (van Roon \& Steenbergen 2006). According to the Arthritis Foundation 1 in 5 adults in the

59 United States are affected by arthritis, indicating a great demand for methods to relieve

60 associated complications (Foundation 2015). A common intervention consists of using

61 increased diameter grip handles on eating utensils. These grips are typically made from a foam-

62 like material and are available in varying sizes such as $3.18 \mathrm{~cm}(1.25 \mathrm{inch})$ and $4.45 \mathrm{~cm}(1.75$

63 inch) diameters as seen in Figure 1. 
Although adaptive utensils with modified handles are commonly used, limited research

65

66

67

68 quantifies the biomechanical effects of larger grips or describes how modified handles affect the ROM of hand joints. Of the prescribed eating and drinking adaptive devices, patients were found to not use $35 \%$ of them (Neville-Jan A 1993). Primary reasons for this noncompliance likely stem from the improper sizing of recommended device (Kraskowsky \& Finlayson 2001; NevilleJan A 1993). An in-depth review of the literature by (Thomas WN 2010) found the four most common reasons for non-compliance for using adaptive equipment are: 1) the patient was not included in deciding on adaptive equipment; 2) inadequate instructions were given; 3 ) the medical condition improves so they no longer need the adaptive equipment; and 4) the patient's environment is favorable to their condition so they no longer need the adaptive equipment. An individualized approach for prescribing assistive equipment that improves the quality of life for clients mirrors the client-centeredness of rehabilitation therapists. A client-centered approach to assistive equipment provision requires client input when deciding on equipment and to ensure its relevance and appropriateness for the client (Hoffmann \& McKenna 2004). Determining the individuals ROM can help with adaptive equipment prescription and may decrease pain associated with simple tasks of daily life and improve utilization and evidence-based rehabilitation outcomes. Bazanski (Bazanski 2010) suggested that a $50^{\circ}$ lack of flexion in metacarpophalangeal joints, the most important joints during grip, causes a $24 \%$ increase in finger impairment. measurement of ROM (Bronner et al. 2010; Carnaz et al. 2013; Piriyaprasarth et al. 2008). One previous study used a biaxial goniometer to analyze thumb movements during the use of hand 
86 held devices, such as mobile phones, and found the electrogoniometer to be both clinically

87 feasible and accurate (Jonsson et al. 2007).

Modified spoon handles can be beneficial while feeding and research has shown positive outcomes regarding the potential benefits of these utensils for patients with conditions including rheumatoid arthritis, Parkinson's disease, and cerebral palsy (Ma et al. 2008; van Roon \& Steenbergen 2006). Handle diameter and its relationship to spoon-use movement was examined in patients with Parkinson's disease. Handles of small $(1.2 \mathrm{~cm})$, medium $(2.0 \mathrm{~cm})$, and large $(3.8$ $\mathrm{cm}$ ) diameter size were studied and the large handles significantly decreased task movement time and subjective scores of comfort and feasibility of use (Ma et al. 2008). This was likely seen as the hand aperture of the participants with Parkinson's disease was significantly smaller than that of the controls. This study provides evidence of the benefits of altering handle size, but accounts for only the overall movement of the hand as a single unit, and does not address how the grip affects individual joints within the hand. arthritis suggests that these assistive devices can help to protect joint integrity by minimizing

101 joint forces and avoiding tight grips (Shipham \& Pitout 2003). Van Roon (2006) examined spoon

102 grip-size and its effects on movement kinematics and food spilling for patients with cerebral

103 palsy. Participants with tetraparesis performed quicker transportation of water from one bowl to

104 another and with less spillage when using a $5 \mathrm{~cm}$ ( 2 inch) diameter modified spoon versus a $3 \mathrm{~cm}$

105 (1.18 inch) and $1 \mathrm{~cm}(0.40$ inch) spoon.

106 While these studies show benefits that may result from using modified spoon handles,

107 they do not study biomechanical changes that occur to individual finger joints when gripping the

108 handles. This study aimed to determine the biomechanical differences in ROM of the fingers 
109 when using three different spoon handles in young healthy subjects. These included a standard

110 spoon, a $3.18 \mathrm{~cm}$ (1.25 inch) diameter modified handle and a $4.45 \mathrm{~cm}$ (1.75 inch) diameter

111 modified handle. These sizes were chosen as they are commonly adopted by patients among

112 those commercially available. The purpose of this study was to determine differences in the

113 ROM required from the joints in the hand when gripping three different sizes of adaptive spoon

114 handles with various diameters.

115

116

117 Materials \& Methods

\section{Subjects}

119 Thirty-four healthy females who were students at the University of Tennessee at Chattanooga, 120 between the ages of 18 and $30(x=20.38 \pm 1.67)$ years of age voluntarily participated in this 121 study. The average grip strength was $58.41 \mathrm{psi}$, consistent with previously published normative

122 values for females between the ages of 20-29 (Bohannon 2006; Peters et al. 2011).

123 Exclusion criteria included previous hand injury, any neurological condition that would

124 impair hand movement, arthritis or any other condition that would prevent the subject from

125 having normal hand function and ROM. To reduce the amount of variables potentially affecting

126 or influencing results, all participants were right handed and only the dominant sides were

127 assessed, as the dominant hand is typically used to grasp utensils. All subjects read and signed an

128 informed consent form in accordance with the Institutional Review Board at the University of

129 Tennessee at Chattanooga (IRB \#14-026). There were no incentives or rewards given for

130 participating. Subjects were recruited using online advertisements sent to students of the

131 University of Tennessee at Chattanooga. 


\section{Equipment}

134 A Jamar hydraulic hand dynamometer ${ }^{\mathrm{a}}$ was used to take a total of 3 measurements of grip

135 strength, which were averaged. The electrogoniometer utilized ${ }^{\mathrm{b}}$ was comprised of an angle

136 display unit and a single axis goniometer with accuracy previously reported as $+/-0.1$ degrees

137 (Christensen 1999). A foam arm rest (Figure 2) was used to provide a comfortable standardized 138 position for the subjects during data collection.

140 Experimental Protocol

141 Subjects were seated with their shoulder in the anatomical position, and their elbow at a 90

142 degree angle, with the hand dynamometer handle placed in the second grip position which is 143 recognized as the standard position for producing the most accurate results (Massy-Westropp et

144 al. 2011; Trampisch et al. 2012). Grip strength was tested by asking participants to maintain a

145 maximal isometric contraction for 3 seconds. Participants then placed their right arm on a foam

146 arm rest to standardize arm position (Figure 2). A single axis electrogoniometer was used to

147 measure the angles created at each joint of the hand (Figure 3).

148

149 For all finger joint measurements subjects were given the three spoons (standard handles, and

150 handle diameter of $3.18 \mathrm{~cm}(1.25 \mathrm{inch})$, and handle diameter of $4.45 \mathrm{~cm}$ (1.75 inch) in

151 randomized order and instructed to grip the spoon as if they were going to feed themselves while

152 keeping all fingers in contact with the spoon. In order to confirm that the subjects maintained a

153 solid grip on the spoon throughout the experiment, a small lightweight object was placed in the

154 spoon to ensure they could lift and balance an object with their grip. Hand measurements 
155 included the distal interphalangeal joint (DIP), proximal interphalangeal joint (PIP), and

156 metacarpophalangeal joint (MCP) for each finger and the interphalangeal (IP) and MCP joint for

157 the thumb. Measurements were obtained for all joints and all fingers by placing one sensor on

158 the proximal bone and one sensor on the distal bone adjacent to the joint being measured (Figure

1593 displays an example of the index finger PIP). The angle was displayed on the display unit and

160 was recorded. All measurements were made in triplicate.

161

\section{Results}

163 Mean values and standard deviations of ROM are reported for each finger, by joint and spoon

164 handle size (Tables 1-5). A 3 x 3 x 4 repeated measures ANOVA (Spoon handle size by Joint by

165 Finger) found main effects on ROM of Joint $\left(F(2,33)=318.68\right.$, Partial $\left.\eta^{2}=.95, p<.001\right)$, Spoon

166 handle size $\left(\mathrm{F}(2,33)=598.73\right.$, Partial $\left.\eta^{2}=.97, \mathrm{p}<.001\right)$, and Finger $(\mathrm{F}(3,32)=163.83$, Partial

$\left.167 \eta^{2}=.94, \mathrm{p}<.001\right)$. Pairwise comparisons showed that as spoon size increased, the range of

168 motion needed decreased in all joints and all fingers $(p<0.01)$. In all five fingers the differences

169 in ROM between the standard spoon and both adaptive spoons was statistically significant $(\mathrm{p}<$

170 0.01), with the adaptive spoons requiring less ROM for grasp. In all five fingers the difference in

171 ROM between the $3.18 \mathrm{~cm}$ (1.25 inch) diameter and $4.45 \mathrm{~cm}$ (1.75 inch) diameter spoons was

172 statistically significant $(\mathrm{p}<0.01)$ with the $4.45 \mathrm{~cm}(1.75 \mathrm{inch})$ diameter spoon requiring less

173 ROM for grasp (Tables 1-5).

175 Discussion

176 This study quantified finger and thumb joint ROM needed for healthy adult females to grip a

177 standard spoon and two different adaptive spoon handle sizes. A statistical comparison between 
178 the ROM for each finger, for each of the three spoons showed a significant difference between

179 the angles formed at each joint, with respect to the spoon handle size. The angle recorded can be

180 thought of as the distance the joint moved from its original position in order to grasp the spoon

181 handle. Joint angles were greater when subjects gripped the standard spoon handle compared to

182 the handles of the modified spoons. The need for greater ROM with a standard spoon indicates a

183 potential challenge for someone with limited hand ROM to grasp a standard sized spoon handle..

184 The variability of the data obtained for hand ROM was actually smaller than expected (Tables 1-

185 5). Some variability between individuals was likely due to the variations in which people grasp a

186 utensil despite standardized instructions being given or holding the spoon The data listed in

187 Tables 1-5 and the statistical analyses confirm less range of motion is required to grip spoons

188 with modified handles. Patients who benefit from the use of such utensils include those

189 diagnosed with conditions that commonly restrict hand ROM, such as patients diagnosed with

190 carpal tunnel, stroke, cerebral palsy, or rheumatoid arthritis (van Roon \& Steenbergen 2006) as

191 well as older adults (Kraskowsky \& Finlayson 2001). Knowing the ROM required by the hand to

192 attain certain grasps may help reduce trial-and-error approach and improve the prescription of

193 ADL utensils and could be a clinically relevant consideration for occupational therapists who

194 often fit patients with such assistive devices.

196 Future Research

197 The aim of this study was to provide quantifiable data to support the common practice of

198 employing adaptive equipment such as spoons with increased handle diameter to reduce ROM

199 required to grip a standard spoon handle and thereby increase independence with feeding

200 activities of daily living. Although this concept was successfully confirmed, different research 
201 hypotheses could be formed and tested using similar methods. For example, information

202 recorded during the data collection process such as measurements of hand size could be

203 investigated to show possible correlations between variables of hand size and the range of

204 motion required to grip the different spoon handle diameters. This would require interpretation of

205 individual results as opposed to the overall group analysis run for this particular study. Advances

206 in biomodeling may present the opportunity to provide custom silverware and other tools based

207 on the individual's hand size, strength, and functional needs. Other variables could be introduced

208 such as questioning the subject for a subjective rating of comfort to establish what may be the

209 ideal handle size as decreased ROM does not necessarily correlate to increased comfort levels or

210 increased efficiency. A more diverse study population including patients with hand deficits likely

211 to use adaptive equipment could be included in future studies. Certain variables such as grip

212 strength may also be a factor in determining the effectiveness of adaptive utensils when the study

213 population has pre-existing hand impairment, as grip strength performance is highly related to

214 the ability of a subject to use their hand functionality

215

\section{Conclusions}

217 The study quantified the hand range of motion needed for adults to use a standard spoon and two

218 commonly available commercial adaptive spoons. It was hypothesized that it would require less

219 range of motion to grip the spoons with modified handles. An electrogoniometer was used to

220 determine range of motion data for 34 healthy subjects. Statistical analysis found significant

221 differences in range of motion between joints and confirmed the hypothesis that less range of

222 motion is required to grip the modified utensils. 


\section{Footnotes}

225 a. Patterson Medical, Warrenville, IL, USA

226 b. Biometrics Ltd, Ladysmith, VA, USA

227

228 
229

230

231

232

233

234

235

236

237

238

239

240

241

242

243

244

245

246

247

248

249

250

251

252

253

254

255

256

257

258

259

260

261

262

263

264

265

266

267

268

269

270

271

272
Bazanski T. 2010. Metacarpophalangeal joint kinematics during a grip of everyday objects using the three-dimensional motion analysis system. Acta Bioeng Biomech 12:79-85.

Bohannon RW. 2006. Hand-held dynamometry: adoption 1900-2005. Percept Mot Skills 103:34.

Brach JS, VanSwearingen JM, Newman AB, and Kriska AM. 2002. Identifying early decline of physical function in community-dwelling older women: performance-based and selfreport measures. Phys Ther 82:320-328.

Bronner S, Agraharasamakulam S, and Ojofeitimi S. 2010. Reliability and validity of electrogoniometry measurement of lower extremity movement. J Med Eng Technol 34:232-242.

Carnaz L, Moriguchi CS, de Oliveira AB, Santiago PR, Caurin GA, Hansson GA, and Coury HJ. 2013. A comparison between flexible electrogoniometers, inclinometers and threedimensional video analysis system for recording neck movement. Med Eng Phys 35:1629-1637.

Christensen HW. 1999. Precision and accuracy of an electrogoniometer. J Manipulative Physiol Ther 22:10-14.

Foundation A. 2015. Arthritis Facts. Arthritis Foundation. http://www.arthritis.org/aboutarthritis/understanding-arthritis/what-is-arthritis.php Available at http://hprints.org/hprints-00714715 (accessed 25 November 2015)

Hoffmann T, and McKenna K. 2004. A Survey of Assistive Equipment Use by Older People following Hospital Discharge. The British Journal of Occupational Therapy 67:75-82.

Jonsson P, Johnson PW, and Hagberg M. 2007. Accuracy and feasibility of using an electrogoniometer for measuring simple thumb movements. Ergonomics 50:647-659.

Kraskowsky LH, and Finlayson M. 2001. Factors affecting older adults' use of adaptive equipment: review of the literature. Am J Occup Ther 55:303-310.

Ma HI, Hwang WJ, Chen-Sea MJ, and Sheu CF. 2008. Handle size as a task constraint in spoonuse movement in patients with Parkinson's disease. Clin Rehabil 22:520-528.

Massy-Westropp NM, Gill TK, Taylor AW, Bohannon RW, and Hill CL. 2011. Hand Grip Strength: age and gender stratified normative data in a population-based study. BMC Res Notes 4:127.

Neville-Jan A PC, Kielhofner G, Davis K. 1993. Adaptive Equipment: A Study of Utilization After Hospital Discharge. Occupational Therapy in Health Care 8:3-14.

Peters MJ, van Nes SI, Vanhoutte EK, Bakkers M, van Doorn PA, Merkies IS, and Faber CG. 2011. Revised normative values for grip strength with the Jamar dynamometer. $J$ Peripher Nerv Syst 16:47-50.

Piriyaprasarth P, Morris ME, Winter A, and Bialocerkowski AE. 2008. The reliability of knee joint position testing using electrogoniometry. BMC Musculoskelet Disord 9:6.

Shipham I, and Pitout SJ. 2003. Rheumatoid arthritis: hand function, activities of daily living, grip strength and essential assistive devices. Curationis 26:98-106.

Thomas WN PL, Gardine CJ. 2010. The Reasons for Noncompliance with Adaptive Equipment in Patients Returning Home After a Total Hip Replacement. Physical and Occupational Therapy in Geriatrics 28:170-180. 
273

274

275

276

277

278

279

280

281

282

283

284

285

286

287

288

289

290

291

292

293

294

295

296

297

298

299

300

301 inch]).

Trampisch US, Franke J, Jedamzik N, Hinrichs T, and Platen P. 2012. Optimal Jamar dynamometer handle position to assess maximal isometric hand grip strength in epidemiological studies. J Hand Surg Am 37:2368-2373.

van Roon D, and Steenbergen B. 2006. The use of ergonomic spoons by people with cerebral palsy: effects on food spilling and movement kinematics. Dev Med Child Neurol 48:888891.

\section{Legend for illustrations}

Figure 1. Adaptive Utensils with Modified Handles. These images, from left to right depict a standard spoon, a spoon with a $3.18 \mathrm{~cm}$ (1.25 inch) diameter handle, and a spoon with a $4.45 \mathrm{~cm}$ diameter handle (1.75 inch).

Figure 2. Foam arm rest to support the forearm.

Figure 3 Single axis goniometer used to measure the Proximal Interphalangeal Joint (PIP) of the index finger. Sensor ' $A$ ' is placed on the intermediate phalanx and sensor ' $\mathrm{B}$ ' is placed on the proximal phalanx of the index finger. (Source: Goniometer and Torsiometer Operating Manual. Biometrics Ltd)

\section{Table legends}

Table 1. Comparison of thumb (first digit) ROM using a standard spoon, and two commercial spoons with enlarged diameter handles $(3.18 \mathrm{~cm}$ [1.25-inch] and $4.45 \mathrm{~cm}$ [1.75-inch]).

Table 2. Comparison of index finger (second digit) ROM using a standard spoon, and two commercial spoons with enlarged diameter handles $(3.18 \mathrm{~cm}[1.25-\mathrm{inch}]$ and $4.45 \mathrm{~cm}[1.75-$ 
302

303 Table 3. Comparison of middle finger (third digit) ROM using a standard spoon, and two

304 commercial spoons with enlarged diameter handles $(3.18 \mathrm{~cm}$ [1.25-inch] and $4.45 \mathrm{~cm}$ [1.75-

305 inch]).

306

307 Table 4. Comparison of ring finger (fourth digit) ROM using a standard spoon, and two

308 commercial spoons with enlarged diameter handles $(3.18 \mathrm{~cm}$ [1.25-inch] and $4.45 \mathrm{~cm}[1.75-$

309 inch]).

310

311 Table 5. Comparison of pinky finger (fifth digit) ROM using a standard spoon, and two

312 commercial spoons with enlarged diameter handles $(3.18 \mathrm{~cm}$ [1.25-inch] and $4.45 \mathrm{~cm}$ [1.75-

313 inch]).

314 


\section{1}

Adaptive Utensils with Modified Handles

Figure 1. Adaptive Utensils with Modified Handles. These images, from left to right depict a standard spoon, a spoon with a $3.18 \mathrm{~cm}$ (1.25 inch) diameter handle, and a spoon with a 4.45 cm diameter handle (1.75 inch). 


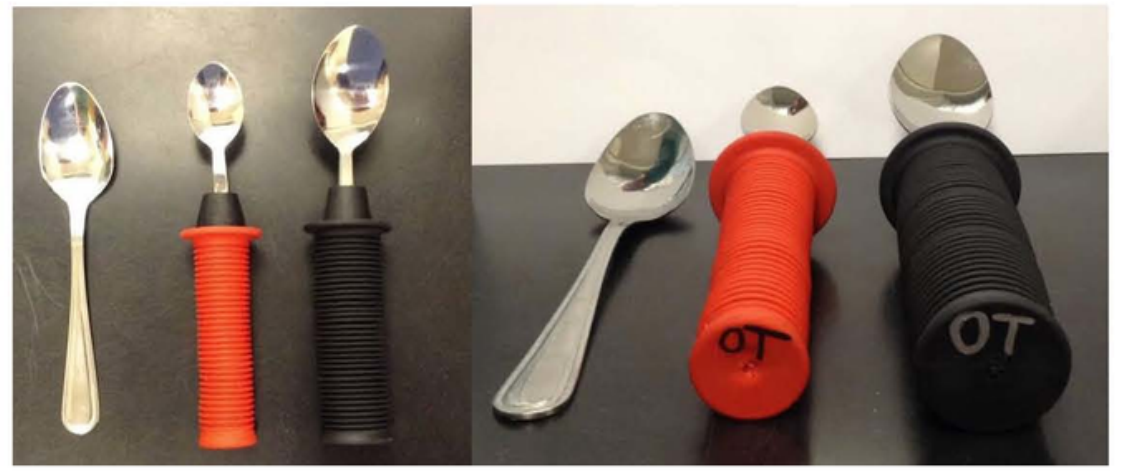


2

Foam arm rest to support the forearm

Figure 2. Foam arm rest to support the forearm. 


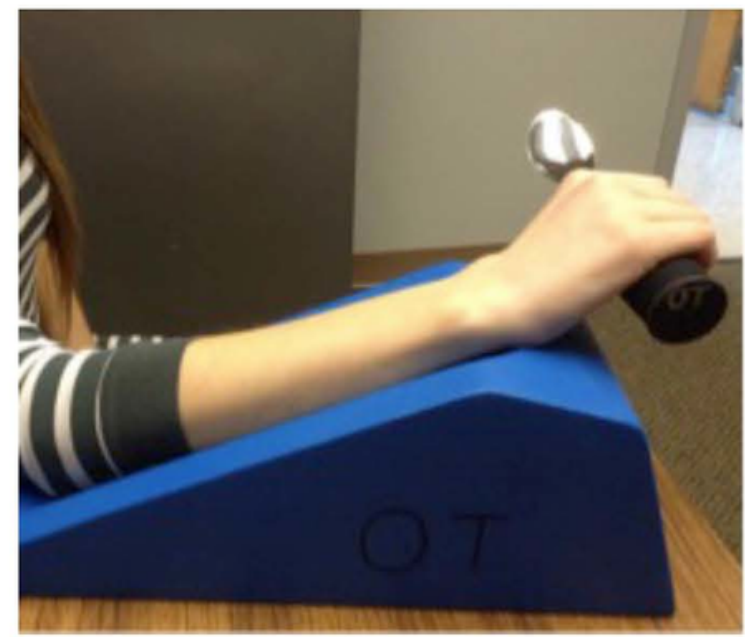




\section{3}

Single axis electrogoniometer

Figure 3 Single axis electrogoniometer. Image is demonstrating measuring the proximal Interphalangeal Joint (PIP) of the index finger. Sensor ' $A$ ' is placed on the intermediate phalanx and sensor ' $B$ ' is placed on the proximal phalanx of the index finger. (Source: Goniometer and Torsiometer Operating Manual. Biometrics Ltd)

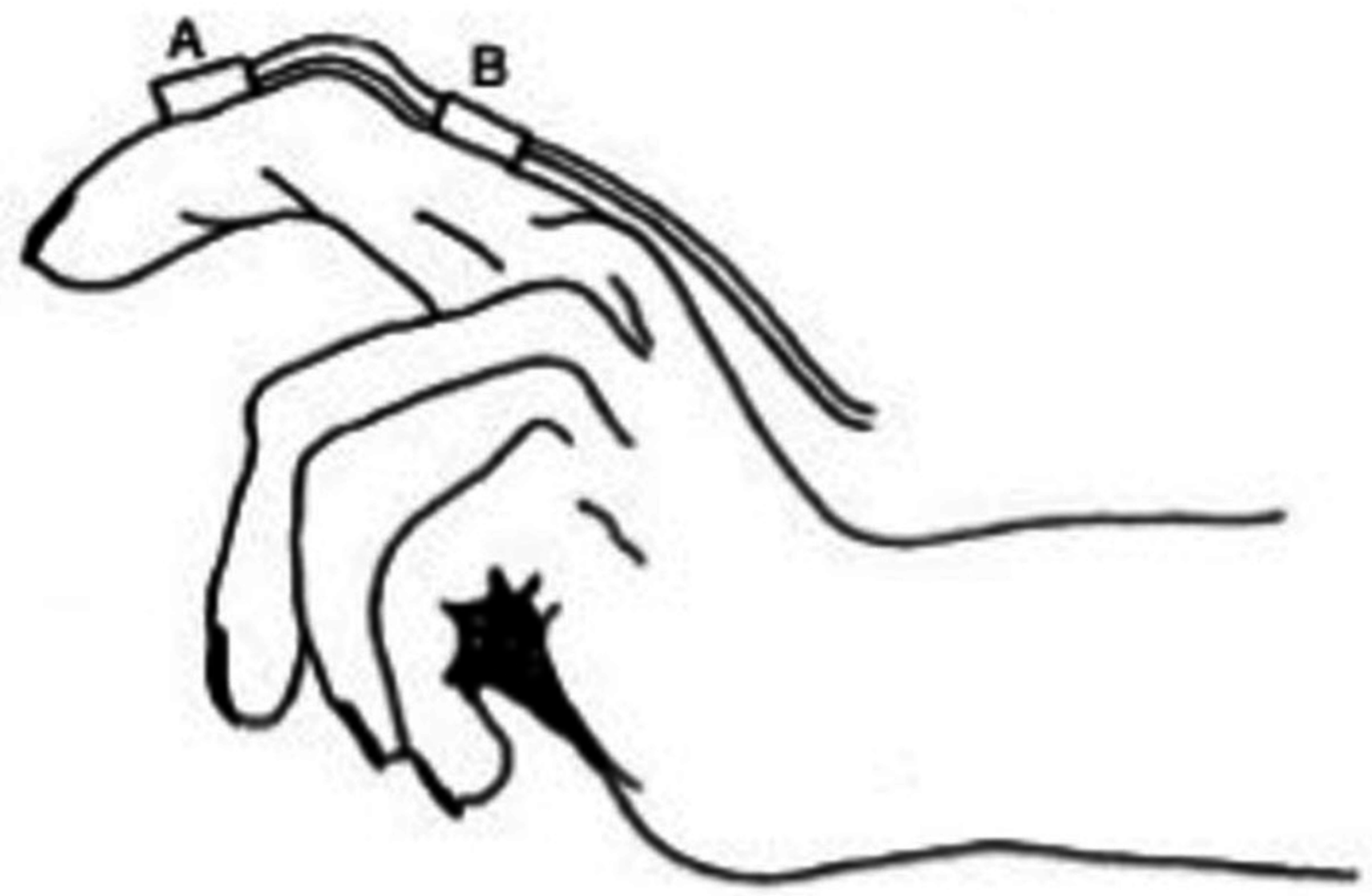




\section{Table $\mathbf{1}$ (on next page)}

Comparison of thumb (first digit) ROM using a standard spoon, and two commercial spoons with enlarged diameter handles (3.18 cm [1.25-inch] and $4.45 \mathrm{~cm}$ [1.75-inch]) 
1 Table 1. Comparison of thumb (first digit) ROM using a standard spoon, and two commercial

2 spoons with enlarged diameter handles (3.18 $\mathrm{cm}$ [1.25-inch] and $4.45 \mathrm{~cm}$ [1.75-inch]).

3

\begin{tabular}{|c|c|c|}
\hline & $\underline{\text { MCP }}$ & $\underline{\text { IP }}$ \\
\hline Standard handle & $30.62^{\circ} \pm 16.08^{\circ}$ & $45.7^{\circ} \pm 19.61^{\circ}$ \\
\hline $\mathbf{3 . 1 8} \mathbf{~ c m}$ & & \\
$\mathbf{( 1 . 2 5}$ inch) handle & $26.46^{\circ} \pm 14.50^{\circ *}$ & $42.28^{\circ} \pm 10.93^{\circ *}$ \\
\hline $\mathbf{4 . 4 5} \mathbf{~ c m}$ & & \\
$\mathbf{( 1 . 7 5}$ inch) handle & $16.53^{\circ} \pm 14.57^{\circ} * \#$ & $36.43^{\circ} \pm 12.13^{\circ} * \#$ \\
\hline
\end{tabular}

4 *Difference between modified handles and standard handle $(\mathrm{P}<0.01)$

5 \#Difference between 3.18 and $4.45 \mathrm{~cm}$ handles $(\mathrm{P}<0.01)$

6

7 
Table 2 (on next page)

Comparison of index finger (second digit) ROM using a standard spoon, and two commercial spoons with enlarged diameter handles $(3.18 \mathrm{~cm}$ [1.25-inch] and $4.45 \mathrm{~cm}$ [1.75-inch]) 
1 Table 2. Comparison of index finger (second digit) ROM using a standard spoon, and two

2 commercial spoons with enlarged diameter handles $(3.18 \mathrm{~cm}$ [1.25-inch] and $4.45 \mathrm{~cm}$ [1.75-

3 inch]).

4

\begin{tabular}{|c|c|c|c|}
\hline & MCP & $\underline{\text { PIP }}$ & DIP \\
\hline Standard handle & $87.47^{\circ} \pm 12.12^{\circ}$ & $106.59^{\circ} \pm 7.70^{\circ}$ & $63.58^{\circ} \pm 11.33^{\circ}$ \\
\hline $\mathbf{3 . 1 8} \mathbf{~ c m}$ & & & \\
$\mathbf{( 1 . 2 5}$ inch) handle & $56.98^{\circ} \pm 13.28^{\circ *}$ & $70.73^{\circ} \pm 6.36^{\circ *}$ & $45.86^{\circ} \pm 6.80^{\circ *}$ \\
\hline $\mathbf{4 . 4 5} \mathbf{~ c m}$ & & & \\
$\mathbf{( 1 . 7 5}$ inch) handle & $40.68^{\circ} \pm 11.77^{\circ} * \#$ & $55.01^{\circ} \pm 8.13^{\circ * \#}$ & $35.59^{\circ} \pm 6.96^{\circ * \#}$ \\
\hline
\end{tabular}

$5 *$ Difference between modified handles and standard handle $(\mathrm{P}<0.01)$

6 \#Difference between 3.18 and $4.45 \mathrm{~cm}$ handles $(\mathrm{P}<0.01)$ 
Table 3 (on next page)

Comparison of middle finger (third digit) ROM using a standard spoon, and two commercial spoons with enlarged diameter handles $(3.18 \mathrm{~cm}$ [1.25-inch] and $4.45 \mathrm{~cm}$ [1.75-inch]) 
1 Table 3. Comparison of middle finger (third digit) ROM using a standard spoon, and two

2 commercial spoons with enlarged diameter handles $(3.18 \mathrm{~cm}$ [1.25-inch] and $4.45 \mathrm{~cm}$ [1.75-

3 inch]).

\begin{tabular}{|c|c|c|c|}
\hline & $\underline{\text { MCP }}$ & $\underline{\text { PIP }}$ & $\underline{\text { DIP }}$ \\
\hline Standard handle & $93.66^{\circ} \pm 10.12^{\circ}$ & $104.53^{\circ} \pm 5.51^{\circ}$ & $71.31^{\circ} \pm 11.01^{\circ}$ \\
\hline $\mathbf{3 . 1 8} \mathbf{~ c m}$ & & & \\
$\mathbf{( 1 . 2 5}$ inch) handle & $67.42^{\circ} \pm 12.89^{\circ *}$ & $67.1^{\circ} \pm 5.78^{\circ *}$ & $50.93^{\circ} \pm 7.07^{\circ *}$ \\
\hline $\mathbf{4 . 4 5} \mathbf{~ c m}$ & & & \\
$\mathbf{( 1 . 7 5}$ inch) handle & $52.98^{\circ} \pm 12.23^{\circ * \#}$ & $53.68^{\circ} \pm 4.94^{\circ} * \#$ & $39.71^{\circ} \pm 7.43^{\circ} * \#$ \\
\hline
\end{tabular}

4 *Difference between modified handles and standard handle $(\mathrm{P}<0.01)$

5 \#Difference between 3.18 and $4.45 \mathrm{~cm}$ handles $(\mathrm{P}<0.01)$

6 
Table 4(on next page)

Comparison of ring finger (fourth digit) ROM using a standard spoon, and two commercial spoons with enlarged diameter handles $(3.18 \mathrm{~cm}$ [1.25-inch] and $4.45 \mathrm{~cm}$ [1.75-inch]) 
1 Table 4. Comparison of ring finger (fourth digit) ROM using a standard spoon, and two

2 commercial spoons with enlarged diameter handles $(3.18 \mathrm{~cm}$ [1.25-inch] and $4.45 \mathrm{~cm}$ [1.75-

3 inch]).

4

\begin{tabular}{|c|c|c|c|}
\hline & MCP & $\underline{\text { PIP }}$ & DIP \\
\hline Standard handle & $81.07^{\circ} \pm 11.79^{\circ}$ & $108.9^{\circ} \pm 5.84^{\circ}$ & $68.17^{\circ} \pm 11.66^{\circ}$ \\
\hline $\mathbf{3 . 1 8} \mathbf{~ c m}$ & & & \\
$\mathbf{( 1 . 2 5}$ inch) handle & $54.89^{\circ} \pm 15.09^{\circ *}$ & $68.05^{\circ} \pm 6.22^{\circ *}$ & $45.98^{\circ} \pm 6.90^{\circ *}$ \\
\hline $\mathbf{4 . 4 5} \mathbf{~ c m}$ & & & \\
$\mathbf{( 1 . 7 5}$ inch) handle & $42.33^{\circ} \pm 14.81^{\circ} * \#$ & $54.82^{\circ} \pm 7.21^{\circ * \#}$ & $33.03^{\circ} \pm 5.02^{\circ * \#}$ \\
\hline
\end{tabular}

$5 *$ Difference between modified handles and standard handle $(\mathrm{P}<0.01)$

6 \#Difference between 3.18 and $4.45 \mathrm{~cm}$ handles $(\mathrm{P}<0.01)$

7

8 
Table 5 (on next page)

Comparison of pinky (fifth digit) ROM using a standard spoon, and two commercial spoons with enlarged diameter handles $(3.18 \mathrm{~cm}$ [1.25-inch] and $4.45 \mathrm{~cm}$ [1.75-inch]) 
1 Table 5. Comparison of pinky (fifth digit) ROM using a standard spoon, and two commercial

2 spoons with enlarged diameter handles (3.18 $\mathrm{cm}$ [1.25-inch] and $4.45 \mathrm{~cm}$ [1.75-inch]).

\begin{tabular}{|c|c|c|c|}
\hline & $\underline{\text { MCP }}$ & $\underline{\text { PIP }}$ & $\underline{\text { DIP }}$ \\
\hline Standard handle & $77.28^{\circ} \pm 19.23^{\circ}$ & $96.08^{\circ} \pm 8.21^{\circ}$ & $75.76^{\circ} \pm 11.04^{\circ}$ \\
\hline $\mathbf{3 . 1 8} \mathbf{~ c m}$ & & & \\
$\mathbf{( 1 . 2 5}$ inch) handle & $51.96^{\circ} \pm 20.83^{\circ *}$ & $51.71^{\circ} \pm 9.39^{\circ *}$ & $39.73^{\circ} \pm 7.49^{\circ *}$ \\
\hline $\mathbf{4 . 4 5} \mathbf{~ c m}$ & & & \\
$\mathbf{( 1 . 7 5}$ inch) handle & $39.06^{\circ} \pm 20.07^{\circ * \#}$ & $42.7^{\circ} \pm 8.76^{\circ * \#}$ & $31.28^{\circ} \pm 9.78^{\circ * \#}$ \\
\hline
\end{tabular}

3 *Difference between modified handles and standard handle $(\mathrm{P}<0.01)$

4 \#Difference between 3.18 and $4.45 \mathrm{~cm}$ handles $(\mathrm{P}<0.01)$ 